\title{
Colonic delivery of nutrients for management of blood glucose in type 2 diabetes patients
}

\author{
Jerzy Szewczyk ${ }^{1}$, JoAnn Giannone ${ }^{1}$, Stefano Marcuard ${ }^{2}$, Tammy Kindel ${ }^{3}$, Patrick Tso ${ }^{4}$, \\ and Roger Nolan ${ }^{1}$
}

${ }^{1}$ BioKier, Inc. Chapel Hill, North Carolina 27514, USA; ${ }^{2}$ Carolina Digestive Diseases; Greenville, North Carolina 27834, USA, ${ }^{3}$ Department of Surgery, Medical College of Wisconsin, Milwaukee, WI 53226, USA, ${ }^{4}$ Department of Pathology and Laboratory Medicine, University of Cincinnati, Cincinnati, $\mathrm{OH} 45237$, USA

Corresponding author: Jerzy Szewczyk, BioKier, Inc. 105 Green Willow Court, Chapel Hill, NC 27514, USA

Submission Date: Aug 9, 2016; Acceptance date: January 27, 2017; Publication date: January 31, 2017

Citation: Szewczyk J, Giannone J., Marcuard S., Kindel T, Tso P, and Nolan R. Colonic delivery of nutrients for management of blood glucose in type 2 diabetes patients. Functional Foods in Health and Disease 2017; 7(1):36-53

\begin{abstract}
Background: It is now widely accepted that bariatric surgeries such as Roux-en-Y gastric bypass (RYGB) and sleeve gastrectomy (SG) can resolve or improve type 2 diabetes mellitus. Postprandial glucagon-like peptide-1(GLP-1) increases after both RYGB and SG and blockade of the GLP-1 receptor suppresses the hypoglycemic effect post-operatively. The expedited delivery of nutrients, including L-glutamine and butyrate, to the distal small intestine and colon, where most GLP-1-secreting enteroendocrine L-cells are expressed, could explain this increase post-surgery. Pharmacological treatments that target nutrient-sensing receptors on L-cells may mimic the effects of bariatric surgeries and may ameliorate deficiencies in gut hormone responses involved in the regulation of glucose and satiety. In this study, we investigated the effects of the colonic delivery of L-glutamine and butyrate on GLP-1 secretion and glucose homeostasis in both a pre-clinical rodent model and clinical type 2 diabetes mellitus (T2DM).
\end{abstract}

Results: Infusion of $4.4 \mathrm{mg}$ of sodium butyrate, compared to saline, into the colon of Zucker diabetic fatty (ZDF) rats increased GLP-1secretion in response to an intra-duodenal glucose challenge. In a chronic study, oral dosing of 40mg of sodium butyrate twice a day, formulated as colon-targeted sustained-release tablets, preserved glucose tolerance and insulin sensitivity in ZDF rats. In ten T2DM patients requiring oral anti-hyperglycemic agents, infusion of $1 \mathrm{~g}$ of L-glutamine into the colon, compared to saline, increased plasma GLP-1 ( $p=0.017$ at $30 \mathrm{~min}$ ) and insulin $(p<0.01$ at $90 \mathrm{~min} ; \mathrm{p}=0.001$ at $120 \mathrm{~min}$; AUC $\mathrm{p}<0.005)$ after an oral glucose challenge. Similar 
infusion with butyrate significantly increased only insulin secretion at $120 \mathrm{~min}$, compared to saline $(p<0.05)$. Neither agent had an effect on glucose disposal.

Conclusion: Targeted colonic delivery of L-glutamine and butyrate augments secretion of mealstimulated GLP-1 and insulin; L-glutamine was more efficacious in humans. Such an approach may be valuable for the management of hyperglycemia in T2DM patients. A chronic clinical study with colon-targeted sustained-release L-glutamine is required to validate this hypothesis.

Key words: Diabetes, nutrients, gut hormones, GLP-1, secretagogues, glucose management.

\section{INTRODUCTION}

It has been widely reported that bariatric surgery developed to treat morbid obesity is very effective in resolving type 2 diabetes mellitus (T2DM). Post-prandial glucagon-like peptide-1 (GLP-1) increases after both Roux-en-Y gastric bypass (RYGB) [1], biliopancreatic diversion (BPD) [2], and sleeve gastrectomy (SG), and blockade of the GLP-1 receptor suppresses the hypoglycemic effect post-operatively [3]. In 2004, a review by Buchwald [4] reported the following: that 83.7 percent of T2DM cases were resolved after surgery; the resolution of T2DM was more successful following gastric bypass surgery than following purely restrictive procedures; and resolution of T2DM often occurred within days of the surgery. In addition to improvement in T2DM, these effects were also observed: 96.9 percent of hyperlipidemia cases were resolved; 75.4 percent of hypertension cases were resolved and 87.1 percent were either resolved or improved; and 61.6 percent of excess weight is lost. In recent years, many articles have reported that an increase in post-prandially released gastrointestinal hormones important in the regulation of glucose and appetite, namely GLP-1 and peptide YY (PYY), coincides with resolution of diabetes following RYGB $[5 ; 6 ; 7 ; 8]$.

The most effective types of procedures in resolving diabetes are RYGB, BPD (biliopancreatic diversion), BPD with duodenal switch, and the sleeve gastrectomy [9]. A common feature of all of these metabolic procedures is expedited delivery of nutrients to the lower gut. It is now widely accepted that expedited delivery of nutrients to the lower gut is responsible for the increased secretion of gut hormones after meals through nutrient-responsive L-cells abundant in the distal small intestine and colon. Besides outperforming traditional anti-diabetes therapies, both oral and injectable, the effect of these metabolic surgeries is long-lasting $[8 ; 10 ; 11 ; 12 ; 13]$.

Several other approaches which also result in expedited delivery of nutrients to the lower gut have been shown to mimic effects of bariatric surgery, including improved gut hormone responses, glucose tolerance, and weight control. Such methods include ingestion of non-digestible carbohydrates, which results in fermentation within the colon that produces short-chain fatty acids [14; 15], ingestion of acarbose inhibitors which prevents digestion of carbohydrates in the upper gut (reviewed by DiNicolantonio et al. [16]), and insertion of an endoscopic duodenal-jejunal bypass liner (endoluminal sleeve) that diverts digestion of food to the lower gut [17]. Although there are side-effects associated with these approaches, making them unsuitable for widespread adoption, their effectiveness illustrates the potential for the development of an oral gut hormone secretagogue therapy with fewer side-effects. The identification of the nutrients responsible for physiologic activation of L-cells has been the focus of many studies and research. Such nutrients 
include long-chain fatty acids [18], short-chain fatty acids [19], and amino acids, of which L-glutamine has been shown to be the most effective [20;21].

The goal of the current study was to identify a natural nutrient that could act as a gut hormone secretagogue when administered to the lower gut and to test the feasibility of developing the nutrient as an oral therapy for the treatment of diabetes. This article reports on the effects of direct delivery of nutrients into the colon on oral glucose-induced gut hormone and insulin secretion in a rat model of diabetes and in T2DM patients, and on the effects of an oral formulation of a model nutrient on glucose tolerance in diabetic rats.

\section{MATERIALS AND METHODS}

\section{Single-Dose $Z$ DF rat study}

We conducted an acute pre-clinical study to investigate the activity of butyrate as a secretagogue for GLP-1 and PYY when administered to the colon of Zucker diabetic fatty (ZDF) rats as a single dose. A $10 \mathrm{mM}$ solution of butyrate was infused via a surgically implanted catheter into the proximal colon in 9-week-old ZDF rats (Charles River). The butyrate infusion was given at the rate of $10 \mathrm{ml}$ over $30 \mathrm{~min}$ based on the report of Plaisancie [22] et al. Five rats received butyrate and four rats received saline. At $15 \mathrm{~min}$ after the start of the butyrate infusion, a glucose challenge of $2 \mathrm{~g}$ was administered through a surgically implanted duodenal catheter. Blood samples were taken from the jugular vein of animals at $-20,-10,5,15,30,60$, and 120 min for analysis of GLP-1 and insulin using the MILLIPLEX MAP Rat Metabolic Hormone Magnetic Bead Panel Metabolism Multiplex Assay by EDM Millipore.

\section{Chronic ZDF Rat Diabetes Prevention Study}

In a second study, butyrate was given orally twice per day for 16 days to 7-week-old ZDF rats (710 weeks of age is the stage of onset of diabetes in ZDF rats). Butyrate was administered as a colon-targeted, slow-release tablet. AAI Pharma Services of Wilmington, NC, manufactured the butyrate tablets using the ProLonic $\mathrm{AQ}^{\mathrm{TM}}$ formulation. ProLonic $\mathrm{AQ}^{\mathrm{TM}}$ releases compounds over the course of several hours in the proximal colon. An appropriate $\mathrm{pH}$-sensitive coating was applied to the tablets to prevent dissolution of tablets and absorption of drug in the upper gut. The profile of release was confirmed by $\mathrm{PK}$ studies in ZDF rats. ZDF rats were randomized into the following groups ( $\mathrm{n}=5 /$ group): placebo, $10 \mathrm{mg}$, or $40 \mathrm{mg}$ butyrate tablets. Basal blood glucose levels were recorded by glucometer every day and averaged over three consecutive days and basal insulin levels were recorded every 7 days.

\section{Single-Dose Study in Diabetes Patients}

Subjects: Ten patients with T2DM (9 females, 1 male), average age 63.1 (range 54-69 years), with $\mathrm{HbAlc}=6.9 \%$, (range $6.1-7.9 \%)$ and average fasting plasma glucose $=135 \mathrm{mg} / \mathrm{dL}$ (range $101-151$ $\mathrm{mg} / \mathrm{dL}$ ) completed the study. Nine subjects were receiving treatment for diabetes during their participation in the study. Five subjects were on monotherapy and four on dual therapy; seven were treated with metformin. The average BMI was $34 \mathrm{~kg} / \mathrm{m}^{2}$ (range $29-47 \mathrm{~kg} / \mathrm{m}^{2}$ ).

Study Protocol: T2DM subjects were treated in a triple-crossover study design. Each subject was administered with the three treatment solutions which were scheduled in a random and blinded 
fashion, at three separate test visits. The study was approved by Western IRB (Puyallup, WA).

Subjects were required to administer an enema solution at 10:00 PM on the evening before each test visit, in addition to fasting overnight prior to each test visit. A second cleansing enema (saline) was administered prior to each test procedure. Test solutions containing L-glutamine (1g), sodium butyrate $(1 \mathrm{~g})$, or placebo (vehicle only) were administered in $100 \mathrm{~mL} 1 \%$ methyl cellulose in saline using a rectal catheter placed in the sigmoid colon. Test solutions were infused for 15 minutes (-18 min to $-3 \mathrm{~min}$ ) and a 50-gram oral glucose challenge was administered at 0 min to patients in a sitting position. Plasma samples taken from a peripheral vein at $-30,-15,5,15,30$, 60, 90, and 120 min were analyzed for glucose, GLP-1, and insulin. GLP-1 and insulin samples were frozen and shipped to BioAgilytix (Durham, NC) for analysis using the Meso Scale Discovery assay technology. Glucose analysis was performed by LabCorp (Burlington, NC).

Preceding the current study, a pilot study in three metformin-naive T2DM patients was conducted following the same procedure as described for the current study. L-glutamine, butyrate, and saline were administered via rectal infusion into sigmoid colon using a random, blinded study design.

\section{Statistical Analyses:}

ZDF Rat Studies: Student's t-test was used to compare the effect of colon-delivered butyrate on hormones. Significance was considered as $\mathrm{p}<0.05$.

Clinical study: Data were analyzed to compare differences in levels of GLP-1 and insulin during an oral glucose tolerance test (OGTT) after dosing of T2DM subjects with L-glutamine, sodium butyrate, or placebo. The AUC were calculated for the entire post-baseline period for each analyte, for each subject, and for each treatment using the trapezoidal rule. Analysis of Variance (ANOVA) was performed on each of the two AUCs (GLP-1 and insulin). Least Square Means (LSMs) and P-values were calculated. Analysis of Variance (ANOVA) appropriate for the study's design (a 3way crossover design) was performed using SAS Version 9.2, PROC MIXED. The ANOVA model included treatment, period, and sequence as effects. The ANOVA model was repeated at each timepoint for both endpoints (GLP-1 and insulin). LSMs and their standard error by treatment, calculated from the ANOVA, were calculated by timepoint. Two-sided P-values based on the ANOVA model were also calculated for the two comparisons of interest (L-glutamine with placebo and sodium butyrate with placebo).

\section{RESULTS}

\section{Single-Dose ZDF Rat Study}

Infusion of butyrate into the colon caused a large secretion of GLP-1 (Fig. 1) and PYY (not shown) into the circulation in response to the glucose load. It also moved the peak (Cmax) of insulin earlier by 15 minutes. GLP-1 secretion was increased in none of four saline-treated rats and in four (4) of five (5) butyrate-treated rats. There was a decrease in the concentration of circulating GLP-1 in the saline group following the infusion, which was presumably due to dilution of basal GLP-1 after absorption of the large volume of vehicle from the colon. There was no difference in the glucose curves between butyrate and saline groups (data not shown). 


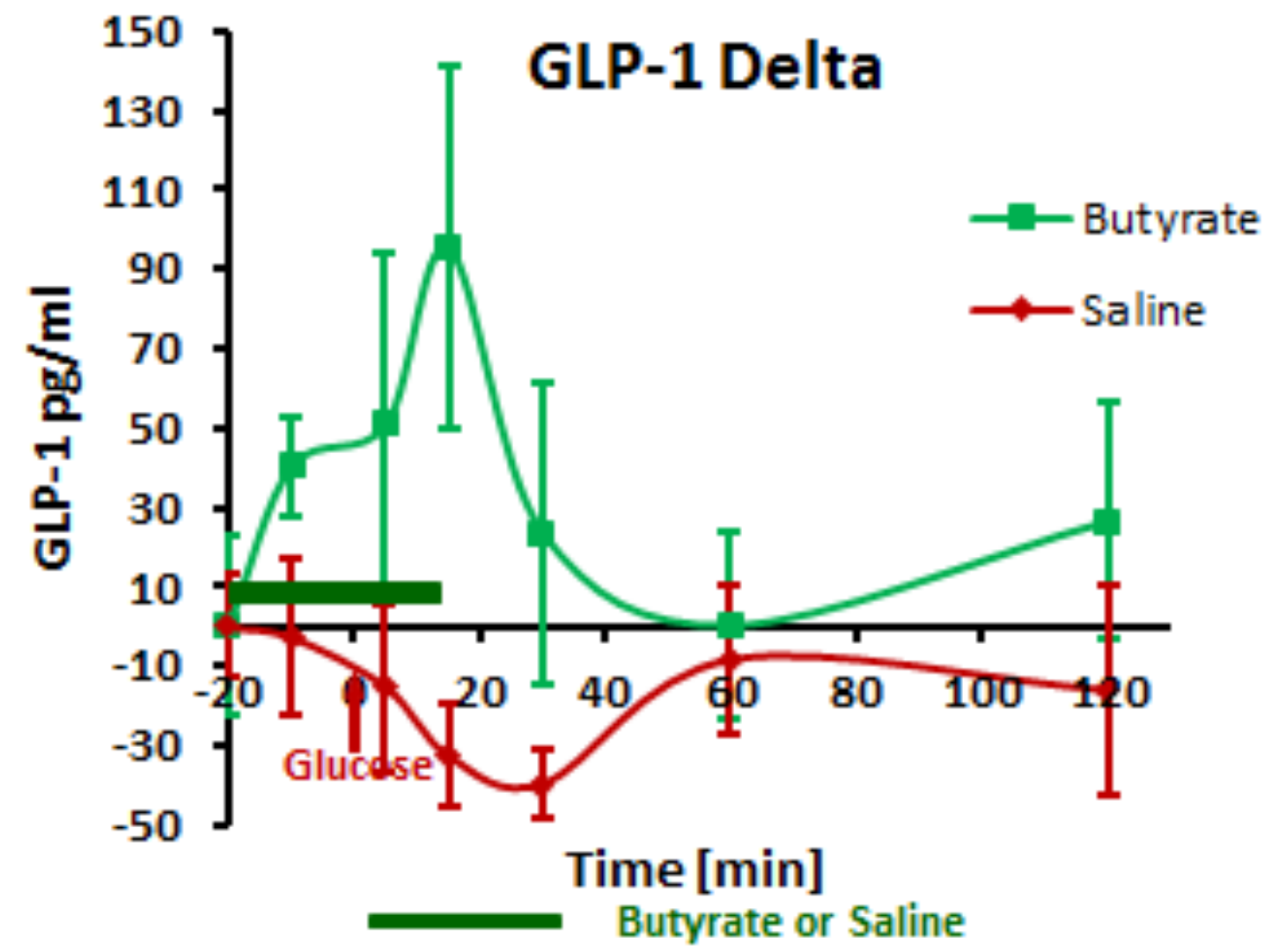

Figure 1: Effect of infusion of butyrate on release of GLP-1 after an oral glucose challenge in ZDF rats. Butyrate was infused from $-15 \mathrm{~min}$ to $15 \mathrm{~min}$ (green bar) and the glucose challenge was given orally at $0 \mathrm{~min}$ (red arrow). Blood samples were collected at the times indicated and analyzed for hormone levels. Because of individual variation, hormone levels are expressed as change from baseline (delta). The difference in peak GLP-1 production between butyrate and saline placebo was statistically significant. Data are expressed as means $\pm \operatorname{SEM}(\mathrm{n}=4$ for saline, $\mathrm{n}=5$ for butyrate).

\section{Chronic ZDF Rat Diabetes Prevention Study}

Colon-targeted butyrate tablets were tested in pharmacokinetic (PK) studies in ZDF rats to validate their release profile. $\mathrm{C}^{13}$-labeled butyrate was used in PK studies to analyze the plasma levels of butyrate released from the tablets. PK analysis showed that butyrate was released from the tablets in the colon over 2 to 4 hours after a 2- to 4-hour delay (data not shown). It was concluded that such a PK profile indicated that butyrate was delivered to the target site in the colon of ZDF rats.

Figure 2 shows that treatment with butyrate twice per day prevented the rise in blood glucose which was seen in the placebo-treated rats ( $\mathrm{p}<0.05$ for all timepoints after $5 \mathrm{~d}$ in the 40 -mg group). Compared to the placebo group, the elevated basal insulin levels in these diabetic rats revealed a trend of decrease during treatment with $10 \mathrm{mg}\left(15^{\text {th }}\right.$ day $)$ and $40 \mathrm{mg}\left(8^{\text {th }}\right.$ and $\left.15^{\text {th }}\right)$ of butyrate, although the difference was not statistically significant (Fig. 3). 


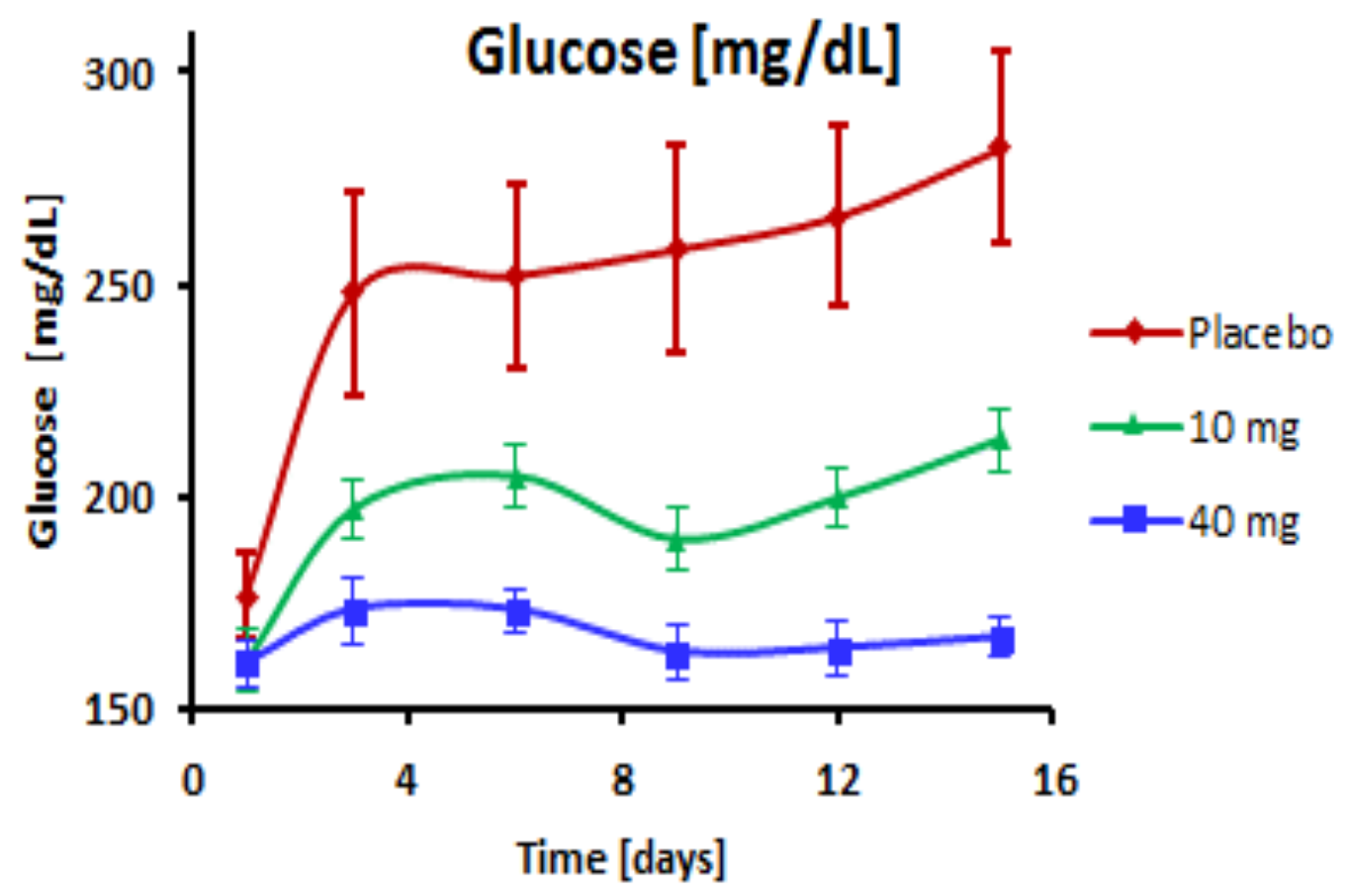

Figure 2: The effect of treatment with colon-targeted slow-release butyrate tablets on basal blood glucose in 7-week old ZDF rats. Rats were dosed orally twice-daily for 16 days and basal blood glucose levels were averaged every 3 days for the duration of the dosing period. Glucose levels in the $40 \mathrm{mg}$ group were statistically different $(\mathrm{p}<0.05)$ to those in the placebo after Day $3(\mathrm{n}=5)$.

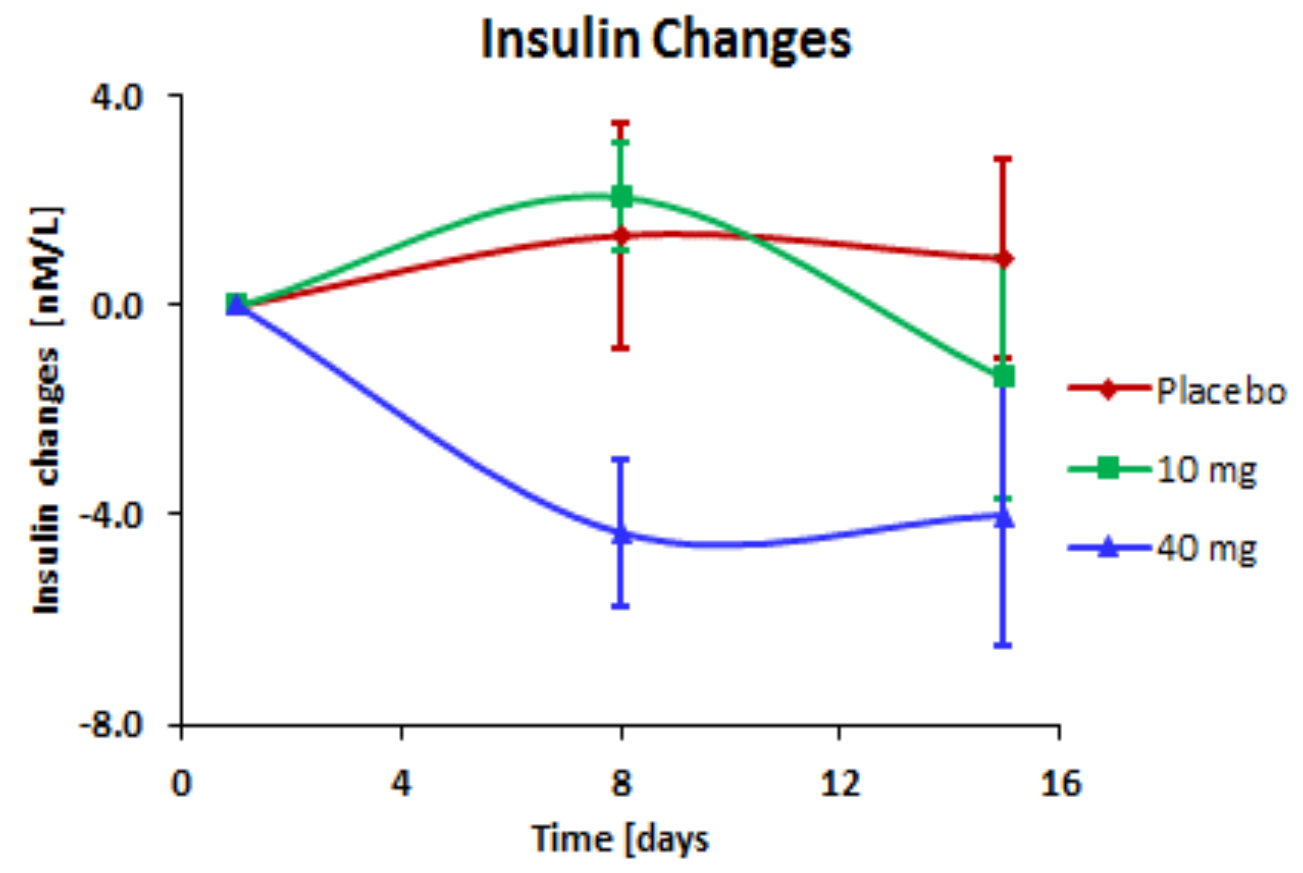

Figure 3: The effect of treatment with colon-targeted slow-release butyrate tablets on basal insulin in 7-week-old ZDF rats. Rats were dosed orally twice-daily for 16 days and basal insulin levels were measured every 7 days for the duration of the dosing period. The y-axis shows the change in insulin levels from the start of dosing. A statistical significant difference in insulin levels between groups was not attained. 
The improvement in insulin sensitivity between placebo and 40mg butyrate groups is demonstrated by calculations of HOMA-IR (Fig. 4). Insulin sensitivity improved in the $40 \mathrm{mg}$ group relative to both placebo and to pretreatment levels ( $\mathrm{p}=0.022$ at day 15$)$.

HOMA-IR

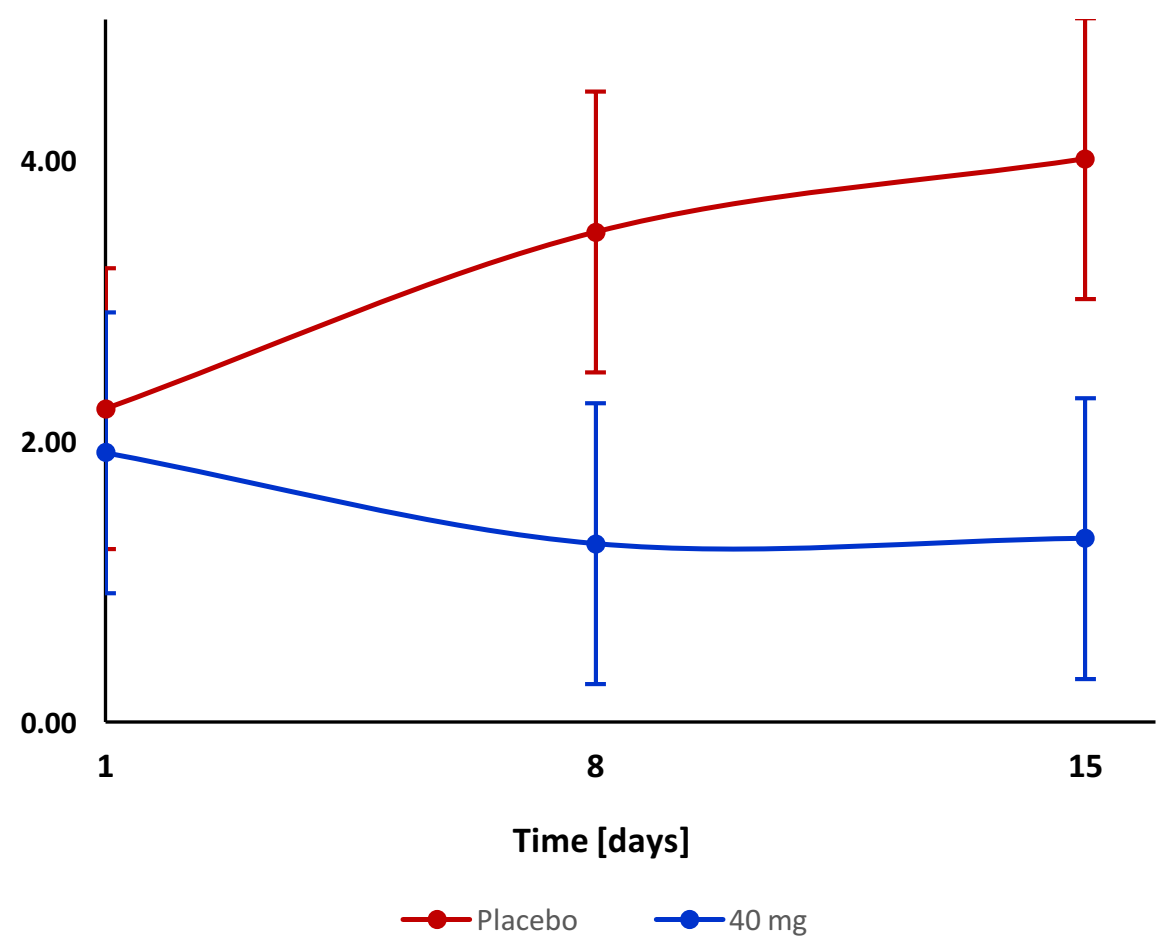

Fig 4: HOMA-IR with colon-targeted slow-release butyrate tablets on basal insulin in 7week-old ZDF rats. Rats were dosed orally twice-daily for 16 days and basal insulin levels were measured every 7 days for the duration of the dosing period. The difference was significant for day 15 $(\mathrm{p}=0.022)$.

\section{Single-Dose Study in Diabetes Patients}

L-glutamine delivered by enema to the sigmoid colon of subjects with T2DM caused an increase when compared to the placebo, in the GLP-1 and insulin responses to an oral glucose challenge. L-glutamine was effective in restoring oral glucose-induced secretion of GLP-1 and insulin to levels approaching those seen in healthy individuals (Figs. 5, 6, and 7). During L-glutamine treatment, GLP-1 levels increased by $3.8 \mathrm{pM}$ at the $30 \mathrm{~min}$ OGTT timepoint, compared to a peak of $2.3 \mathrm{pM}$ at $15 \mathrm{~min}$ for the placebo group (Fig. 5). At this timepoint, the GLP-1 mean change for L-glutamine was statistically significantly different from that of placebo $(\mathrm{P}=0.0176)$. There was no significant difference in GLP-1 between placebo and butyrate treatments. L-glutamine infusion significantly increased insulin levels at $90 \mathrm{~min}(\mathrm{P}=0.0081)$ and $120 \mathrm{~min}(\mathrm{P}=0.0010)$ after the glucose challenge, compared to placebo (Fig 6). There was a statistically greater mean insulin response to butyrate than placebo at $120 \mathrm{~min}$ after glucose challenge ( $\mathrm{P}=0.0449$; Fig. 6). For the AUC of insulin favoring L-glutamine over placebo there was a statistically significant greater increase in $\left(\mathrm{AUC}_{0-120 \mathrm{~min}}: 2614.9\right.$ vs $\left.1756.6 \mu \mathrm{U} / \mathrm{mL} / \mathrm{min}, \mathrm{P}=0.037\right)$. There was no difference in AUC for insulin between butyrate and placebo. 


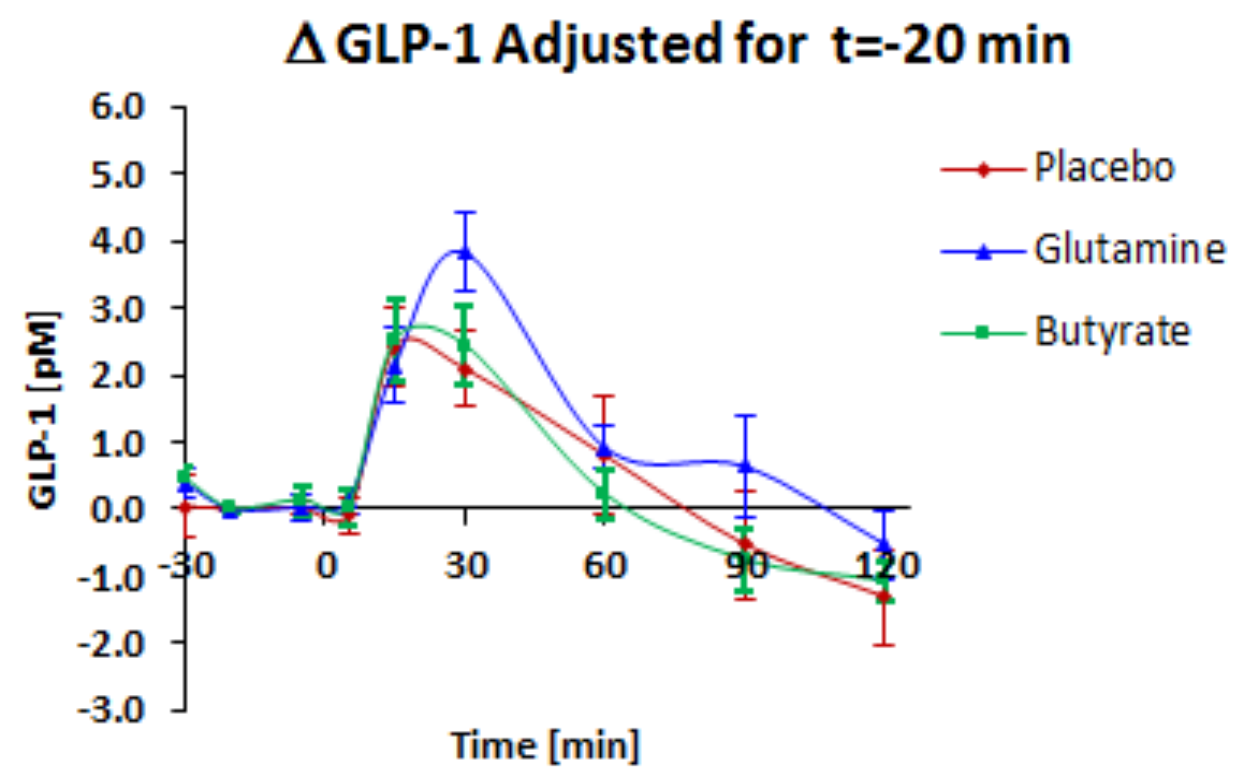

Figure 5: Changes in total GLP-1 over time (min). Diabetic subjects $(n=10)$ were administered an OGTT in the sitting position following infusion into the colon of either L-glutamine (1g), sodium butyrate $(1 \mathrm{~g})$, or placebo (vehicle, $100 \mathrm{~mL} 1 \%$ methyl cellulose in saline) in random order, on separate occasions. The curves, smoothed by spline interpolation, show net circulating total GLP-1 levels, relative to baseline $(\mathrm{t}=-20 \mathrm{~min})$, over the course of the OGTT (120min).

There was no effect of L-glutamine on gut hormones and insulin in the absence of an oral glucose challenge (not shown).

\section{Adjusted for baseline insulin secretion}

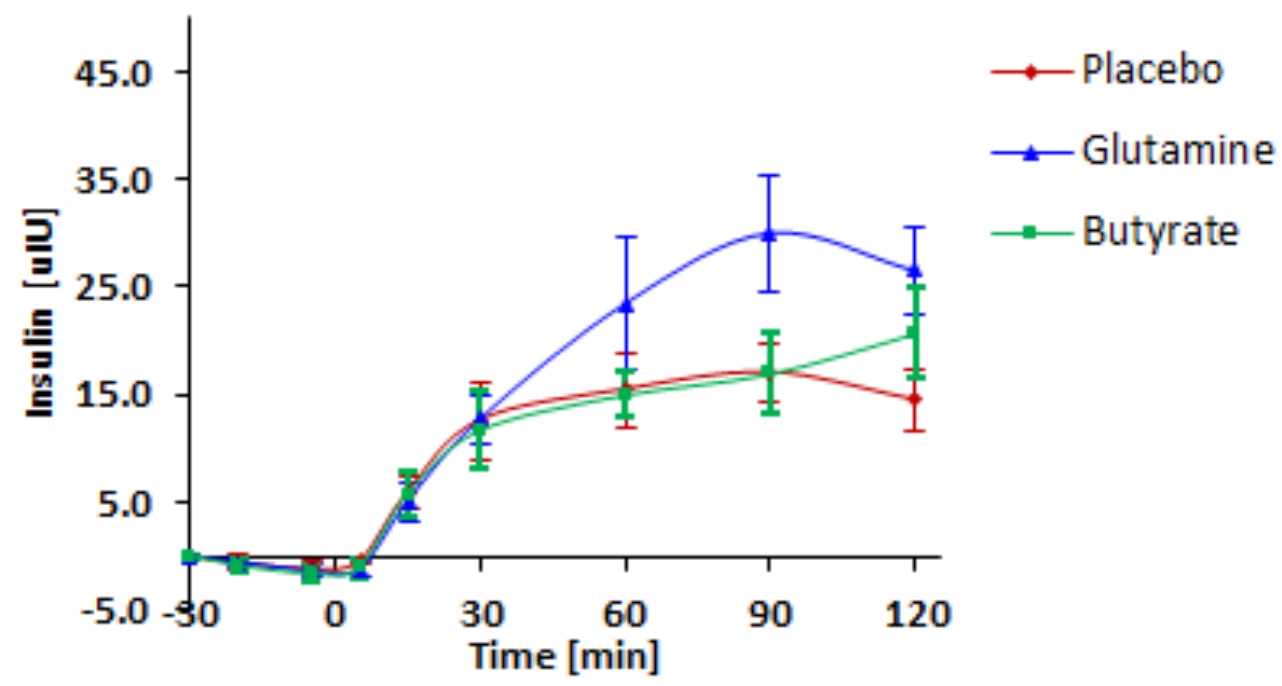

Figure 6: Changes in insulin values over time (min). Diabetic subjects $(n=10)$ were administered an OGTT following infusion into the colon of either L-glutamine (1g), sodium butyrate (1g), or placebo in random order, on separate occasions. The curves, smoothed by spline interpolation, show net circulating insulin levels, relative to baseline ( $\mathrm{t}=-30 \mathrm{~min})$, over the course of the OGTT $(120 \mathrm{~min})$. 
The observed increases in both GLP-1 and insulin after a single dose of $1 \mathrm{~g}$ of intracolonic Lglutamine approached the levels seen in healthy non-diabetic subjects (Fig.7). However, the fact that healthy volunteers received $70 \mathrm{~g}$ of oral glucose while diabetes subjects received only $50 \mathrm{~g}$ for safety reasons also needs to be taken into account.
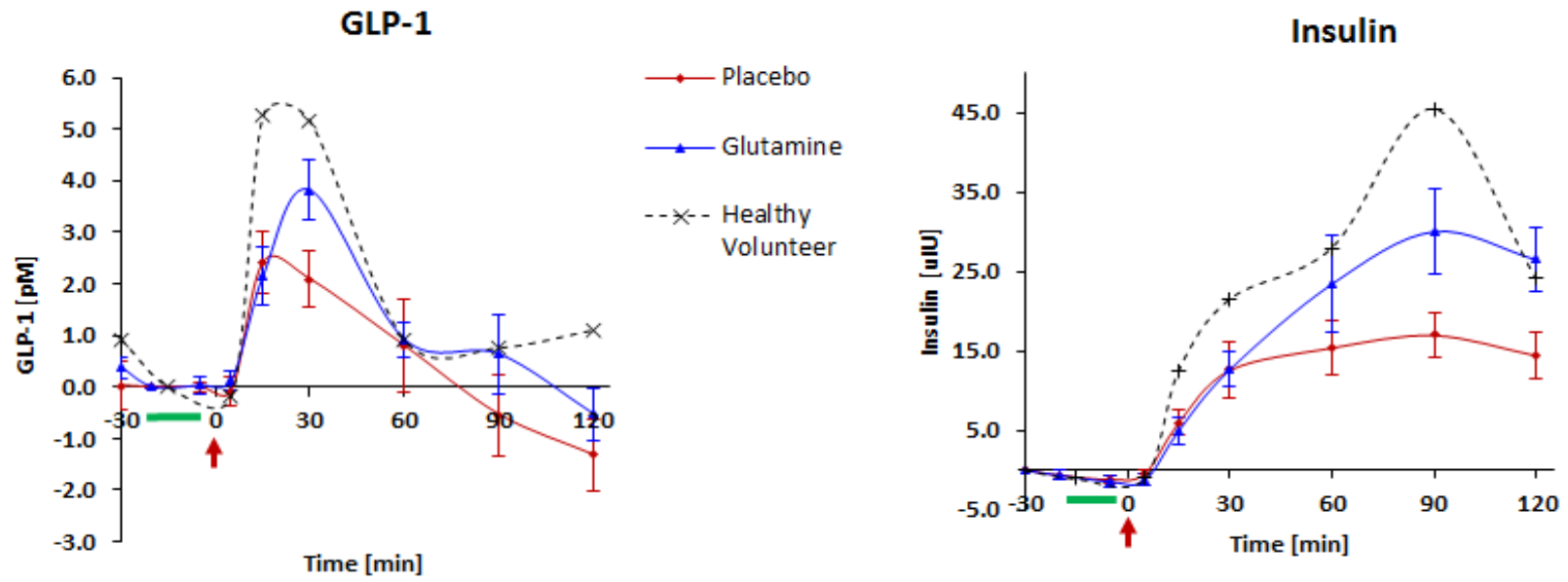

Figure 7: The effect of infusion into the sigmoid colon of L-glutamine on oral glucoseinduced GLP-1 and insulin secretion in T2DM patients: comparison with normal responses. L-glutamine (blue) or saline (red) was infused for 15 minutes (green bar) and the glucose challenge was administered at $0 \mathrm{~min}$ (red arrow); hormones were measured in peripheral blood samples collected over $120 \mathrm{~min}$. Data are shown as change from pretreatment levels $(\mathrm{n}=10)$. Responses from a typical healthy non-diabetic subject are shown for comparison (diamonds). Differences between L-glutamine and placebo are statistically different for GLP-1 at 30 min and for insulin at 90 and $120 \mathrm{~min}$. Differences in insulin AUC for L-glutamine and placebo are statistically significant.

The only effect of butyrate was an increase in insulin secretion at $120 \mathrm{~min}$. In a previous exploratory (pilot) study, we examined the effects of butyrate and L-glutamine in drug-naïve diabetes patients in which both agents greatly augmented the secretion of GLP-1 and insulin and caused a left-shift in the insulin secretion curve (Fig.8). No statistically significant differences were observed in the pilot study of three subjects.

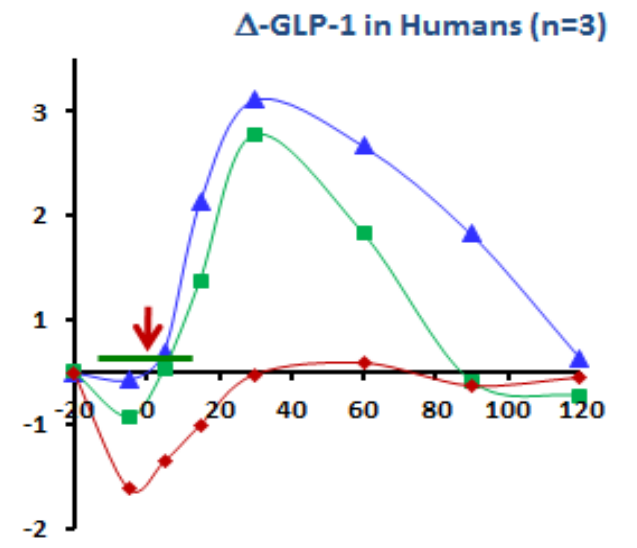

Insulin Changes in Humans ( $n=3)$

Nutrient or Placebo
Glucose

Figure 8: The effect of infusion into the sigmoid colon of L-glutamine and butyrate on oral glucose-induced GLP-1 and insulin secretion in drug-naive T2DM patients. For GLP-1 data are shown as change from pretreatment levels $(n=3)$. 
Although effects of L-glutamine and butyrate on acute GLP-1 and insulin responses to an oral glucose challenge were observed, there was no effect of the test agents on plasma glucose during the time-course of the OGTT (data not shown). In all three treatment groups, plasma glucose peaked between 60 and $90 \mathrm{~min}$.

\section{DISCUSSION}

The series of preclinical and clinical studies presented in this paper investigated whether administration of individual nutrients to the colon in diabetic subjects could reproduce the effects of metabolic surgery on restoration of gut hormone secretion and thereby represent a potential approach for the treatment of diabetes.

ZDF rats were used to test the concept that direct delivery of a nutrient with known gut hormone secretagogue activity to the colon was able to augment postprandial secretion of gut hormones and insulin. Based on its known activity in rats, butyrate was used as the test compound in rats and was shown in single doses to augment secretion of GLP-1 and PYY, with a left-shift in insulin secretion. A coincident effect on all three parameters is significant, as GLP-1 secretion after meals is accompanied by co-secretion of PYY, oxyntomodulin, and GLP-2, while also being a stimulus for insulin secretion [23]. These effects represent those seen in non-diabetic rats. Furthermore, something else relevant to these results is the much-published finding that increased secretion of both GLP-1 and PY, along with the normalization of the insulin curve in response to an oral glucose challenge, accompanies the resolution of diabetes following Roux-en-Y gastric bypass surgery [5].

To test whether butyrate dosed orally could have an effect on glucose tolerance, ZDF rats were dosed with colon-targeted, sustained-release butyrate tablets for 16 days during the period when they spontaneously develop diabetes. Butyrate dose-dependently prevented the development of diabetes, while also demonstrating a tendency to improve insulin sensitivity, a hallmark of gastric bypass surgery in diabetic rats [24] and humans [25]. The effect on basal blood glucose compares very favorably with the effects reported in studies of drugs from the class of DPP-IVinhibitors, which block GLP-1 degradation, and through stable GLP-1 analogs [26; 27]. A beneficial effect on insulin sensitivity is key to an efficacious treatment, as insulin resistance is instrumental in the development of T2DM. A desirable component of a treatment for T2DM is improvement in insulin sensitivity.

Once a preclinical proof of the concept was demonstrated, studies investigating whether this concept could be reproduced in humans were conducted. In a comparative study trying to identify the relative activities of secretagogues in humans, subjects with T2DM were administered single short infusions to the sigmoid colon of nutrients to investigate the effects on oral glucose-induced GLP-1 secretion, insulin secretion, and glucose disposal. In addition to butyrate, the effects of Lglutamine were investigated in the clinical studies because it has recently been shown to have strong gut hormone secretagogue activity in humans $[21 ; 28]$. In a sample of T2DM patients who were representative of the diabetes population (i.e., those treated with one to three anti-diabetes drugs, mostly including metformin), L-glutamine was shown to have better efficacy than butyrate in increasing both GLP-1 and insulin responses to an oral glucose challenge. The improvement in GLP-1 and insulin responses resembled those in healthy, nondiabetic individuals and in diabetes subjects after metabolic surgeries, including RYGB [25; 29]. In this study, only $1 \mathrm{~g}$ of L-glutamine 
was needed to evoke such effects when delivered to the colon. This starkly contrasts attempts to increase GLP-1 secretion using unformulated oral L-glutamine. In Samocha-Bonet's publication [28], 30g of oral L-glutamine was required to increase GLP-1 secretion in response to a mixedmeal tolerance test to a similar degree as $1 \mathrm{~g}$ of L-glutamine administered directly to the colon. It can be deduced that L-glutamine targeted to the colon is much more effective than L-glutamine administered orally, which is to be expected since amino acids are largely absorbed in the upper gut [30].

The significance of the increase in circulating GLP-1 levels after L-glutamine administration to T2DM subjects is supported by the subsequent increase in circulating insulin levels. GLP-1 controls blood glucose at least in part through regulation of glucose-dependent insulin secretion [23]. The lag between peak GLP-1 and insulin appearance in the circulation is approximately 60 min in this study; peak GLP-1 levels after L-glutamine dosing occurs at 30 min and peak insulin levels at $90 \mathrm{~min}$. This is in accordance with the findings of Samocha-Bonet et al., who demonstrated that after dosing with oral L-glutamine, postprandial GLP-1 levels peaked at 15-30 min and insulin at 60min [28; 31]. Recently, Dr. Gribble's group demonstrated that a single oral dose of encapsulated ileal-release glutamine can promote increased secretion of GLP-1 and is associated with increased insulin release [32;33], while the effect of ileal-delivered glutamine was relatively small. Dr. Gribble's single-dose studies confirm that glutamine can have a positive effect on GLP-1 and insulin responses when directed to the lower gut. No effect of single-dose glutamine on glycemia was observed in the Gribble studies, which is in accordance with the findings in this current study. However, studies on bariatric surgery [34] and on administration of propionate to the colon [35] suggest that chronic improvement of GLP-1 secretion will improve insulin sensitivity and glucose tolerance, in addition to reducing weight. Therefore, it is anticipated that the chronic treatment of the colon with L-glutamine, a known gut hormone secretagogue, will have a clinically significant effect on glucose tolerance.

L-glutamine administration to the colon of T2DM subjects had no effect on the secretion of gut hormones and insulin in the absence of an oral glucose challenge. The finding in this study that $1 \mathrm{~g} \mathrm{~L}$-glutamine did cause an increase in the secretion of GLP-1 and insulin in response to an OGTT in this study implies that L-glutamine works in concert with the ingestion of glucose to stimulate the secretion of GLP-1. The potential action of L-glutamine to prime L-cells to respond to subsequent meals would be a useful property of a potential therapeutic for T2DM.

The magnitude of the effect of L-glutamine on GLP-1 is not large but appears to be clinically significant, approaching the GLP-1 response seen in non-diabetic subjects. In a published study, pharmacological inhibition of DPP-IV that leads to significant improvement in glucose regulation resulted in increases of circulating postprandial levels of GLP-1 of 1.5- to 3-fold [36], which are comparable to the increases seen in this study with colonic L-glutamine treatment. The conclusion from review of many reported studies is that the dynamic range of plasma GLP-1 levels is relatively small. There are several reasons for the finding that the increases in GLP-1 in the peripheral circulation are of relatively small magnitude. A physiologically significant factor is that GLP-1 acts as a local hormone in the gut, before it is diluted in the systemic circulation, meaning that even peak levels measured in peripheral samples are low. The intestinal mucosa and hepatic portal vein are densely innervated with afferent nerves that respond to gut hormones $[37 ; 38]$. Inhibition of these GLP-1 receptors in the portal vein was shown to reduce glucose clearance [39]. 
The findings that half of the GLP-1 is inactivated by DPP-4 before it reaches the circulation and that intestinal lymph has 10 to 20-fold higher concentrations of GLP-1 than portal plasma indicate that GLP-1 does not act via a purely endocrine mechanism [40]. In fact, GLP-1 has a plasma halflife of $1-2 \mathrm{~min}$ [41;42]. Additionally, it is relevant that there is feedback inhibition of GLP-1 secretion, which contributes to keeping circulating levels of GLP-1 low. Somatostatin released from the a-cells of the pancreas in response to GLP-1 secretion has been shown to block further secretion of GLP-1 from L-cells in the lower gut [43].

Surprisingly, there was a muted effect of butyrate on the acute biomarker responses in this single-dose clinical study. This is somewhat unexpected, especially considering the known effects of butyrate produced in the colon by fermentation of fiber and saccharides on glucose regulation $[15 ; 44]$. The effects of acute treatment on gut hormone secretion in the animal studies are described in this article and in other model systems [45]. However, this may be due to different experimental conditions in this study. Another explanation is that participants in this study remained on anti-diabetes medications (mostly metformin which is known to increase basal GLP1 levels and blunt further increases [46]) during the course of the study, which may have masked an effect of butyrate and blunted an effect of L-glutamine. Evidence that can support this hypothesis is an earlier pilot study conducted by the authors, which demonstrated that butyrate, in addition to L-glutamine, had a large effect on oral glucose-induced secretion of GLP-1 and insulin in anti-diabetes medication-naïve T2DM subjects. It is worth noting that basal levels of GLP-1 in the metformin-naïve subjects were considerably lower than in metformin-treated subjects. In accordance with the lack of effect of butyrate on acute GLP-1 secretion in this study, there is no evidence in the literature to support the concept that chronic effects of butyrate on glucose tolerance are correlated with measurable acute effects of butyrate on GLP-1 secretion. While a 12month treatment of diabetic patients with tagatose, which is fermented to butyrate in the colon, demonstrated a beneficial effect on HbA1c (lowering by 1\%) [44], single doses of tagatose did not increase GLP-1 [47]. This further highlights the difficulty in demonstrating measurable effects on oral glucose-induced GLP-1 secretion, even after treatment which delivers butyrate to the colon in levels that improve glucose tolerance. One hypothesis is that the statistically significant effect of L-glutamine on oral glucose-stimulated GLP-1 and insulin secretion in this single-dose clinical study predicts a much better effect of L-glutamine in improving glucose tolerance after chronic administration than that observed with butyrate, provided via fermentation, in the tagatose studies.

In regards to the lack of effect of the test agents on plasma glucose during the OGTT, we conclude that the duration of sample collection was too short to see any effect on glucose. In this study, insulin levels were higher for L-glutamine vs. placebo only after $90 \mathrm{~min}$, at which time the plasma glucose had already peaked and was decreasing. Given the expected lag (60-90 min) between elevating insulin levels and observing its effect on glucose lowering, a full effect on blood glucose would not be seen till $150 \mathrm{~min}$ to $180 \mathrm{~min}$ after the glucose challenge, as demonstrated by Nauck's group [48], which is outside the observation range in this study. Notably, there was an expected lag between peak GLP-1 secretion (at approximately $30 \mathrm{~min}$ ) and peak insulin secretion (at approximately $90 \mathrm{~min}$ ) in this study. The lack of effect of acute dosing with gut hormone secretagogues on glucose levels, while unexpected, is not unprecedented. Chang et al. [49] reported that 7.5 and $15 \mathrm{~g}$ of intraduodenal glutamine did not affect plasma glucose after a duodenal glucose challenge while significantly increasing GLP-1 levels. We observed similar 
results after intracolonic infusion of butyrate in ZDF rats. A single dose of $4.4 \mathrm{mg}$ of butyrate combined with a glucose challenge significantly increased GLP-1 secretion, while there was no acute effect on plasma glucose. Despite this, repeated dosing of colon-targeted butyrate (16 days) resulted in full prevention of diabetes in the rat model while improving insulin sensitivity (Fig 4). While in this study there was no acute effect of L-glutamine on glucose, chronic treatment with a gut hormone secretagogue would be expected to result in lowering of both fasting and postprandial glucose, as observed after gastric bypass surgery and chronic treatment with fermentable carbohydrate.

\section{CONCLUSIONS}

The series of studies reported in this article demonstrate that the administration of individual nutrients which are known to be physiologic gut hormone secretagogues to the colon of diabetic individuals is able to augment the secretion of gut hormones, including GLP-1, and insulin in response to an oral glucose challenge. Additionally, these studies show that one of the nutrients, in an oral formulation for the targeted, sustained release in the colon of diabetic rats when dosed chronically can have a profound effect on diabetes. The comparative study of butyrate and Lglutamine was conducted in humans to eliminate any species effects that may skew the selection of lead candidate for development in the clinic. This study confirmed that gut hormone secretagogues such as L-glutamine are active in small amounts when administered to the colon, and thereby may represent a novel pharmacological approach by targeting the nutrient-sensing receptors in the lower gut to ameliorate the defective in glucoregulatory and satiety mechanisms in diabetes, and possibly in pre-diabetes.

It was also confirmed that L-glutamine is a suitable lead compound to formulate for oral delivery for future clinical studies. The authors plan to investigate the effects of chronic dosing in T2DM subjects with oral L-glutamine formulated for sustained release in the colon. Reported studies demonstrating the anti-diabetes effects of similar amounts of butyrate released in the colon over a similar time-course during fermentation of non-digestible carbohydrates [14] suggest that an oral formulation of L-glutamine could be effective, but without the side-effects of fermentation.

List of Abbreviations: RYGB - Roux-en-Y gastric bypass; BPD - biliopancreatic diversion; GLP-1 - glucagon-like peptide-1; PYY - peptide YY; ZDF - Zucker diabetic fatty; ANOVA - Analysis of Variance;LSM - Least Square Mean; PK - pharmacokinetic; OGTT - oral glucose tolerance test; DPP-IV - dipeptidyl peptidase-IV

Competing Interests: All research included in this article was funded by BioKier, and salary of J. Szewczyk, J. Giannone and R. Nolan were paid by BioKier

Author's Contributions: All authors contributed to this study.

Acknowledgments and Funding: We would like to thank Jazett Shivers and Katrina Strickland for assistance with execution of this study, and Gianna Preston for assisting with study material preparation and data entry. 


\section{REFERENCES}

1. Salehi M., Prigeon, R. L., and D'Alessio, D. A. 2011. Gastric bypass surgery enhances glucagon-like peptide 1-stimulated postprandial insulin secretion in humans. Diabetes. 60: $2308-2314$.

2. Astiarraga B., Gastaldelli, A., Muscelli, E., Baldi, S., Camastra, S., Mari, A., Papadia, F., Camerini, G., Adami, G., Scopinaro, N., and Ferrannini, E. 2013. Biliopancreatic diversion in nonobese patients with type 2 diabetes: impact and mechanisms. J.Clin.Endocrinol.Metab. 98: 2765 - 2773.

3. Salehi M., Gastaldelli, A., and D'Alessio, D. A. 2014. Blockade of glucagon-like peptide 1 receptor corrects postprandial hypoglycemia after gastric bypass. Gastroenterology. 146: $669-680$.

4. Buchwald H., Avidor, Y., Braunwald, E., Jensen, M. D., Pories, W., Fahrbach, K., and Schoelles, K. 10-13-2004. Bariatric surgery: a systematic review and meta-analysis. JAMA. 292: 1724 - 1737.

5. Morinigo R., Moize, V., Musri, M., Lacy, A. M., Navarro, S., Marin, J. L., Delgado, S., Casamitjana, R., and Vidal, J. 2006. Glucagon-like peptide-1, peptide YY, hunger, and satiety after gastric bypass surgery in morbidly obese subjects. J.Clin.Endocrinol.Metab. 91: $1735-1740$.

6. Karamanakos S. N., Vagenas, K., Kalfarentzos, F., and Alexandrides, T. K. 2008. Weight loss, appetite suppression, and changes in fasting and postprandial ghrelin and peptideYY levels after Roux-en-Y gastric bypass and sleeve gastrectomy: a prospective, double blind study. Ann.Surg. 247: 401 - 407.

7. Stratis C., Alexandrides, T., Vagenas, K., and Kalfarentzos, F. 2006. Ghrelin and peptide YY levels after a variant of biliopancreatic diversion with Roux-en-Y gastric bypass versus after colectomy: a prospective comparative study. Obes.Surg. 16: 752 - 758.

8. Laferrere B., Heshka, S., Wang, K., Khan, Y., McGinty, J., Teixeira, J., Hart, A. B., and Olivan, B. 2007. Incretin levels and effect are markedly enhanced 1 month after Rouxen-Y gastric bypass surgery in obese patients with type 2 diabetes. Diabetes Care. 30: $1709-1716$.

9. Segal-Lieberman G., Lang, A., Lahav, M., Lieberman, N., Paster, A., Konvalina, N., Kanety, H., Hemi, R., Ilani, J., and Cohen, O. 2015. Acute Effects of Endo-barrier Treatment on Glucose Homeostasis in Obese Uncontrolled Diabetic Subjects -967-P. Diabetes. 64: A235 - A382.

10. Brethauer S. A., Aminian, A., Romero-Talamas, H., Batayyah, E., Mackey, J., Kennedy, L., Kashyap, S. R., Kirwan, J. P., Rogula, T., Kroh, M., Chand, B., and Schauer, P. R. 2013. Can diabetes be surgically cured? Long-term metabolic effects of bariatric surgery in obese patients with type 2 diabetes mellitus. Ann.Surg. 258: 628 - 636.

11. Pories W. J., Swanson, M. S., MacDonald, K. G., Long, S. B., Morris, P. G., Brown, B. M., Barakat, H. A., deRamon, R. A., Israel, G., Dolezal, J. M., and . 1995. Who would have thought it? An operation proves to be the most effective therapy for adult-onset diabetes mellitus. Ann.Surg. 222: 339 - 350.

12. Sjostrom L., Gummesson, A., Sjostrom, C. D., Narbro, K., Peltonen, M., Wedel, H., 
Bengtsson, C., Bouchard, C., Carlsson, B., Dahlgren, S., Jacobson, P., Karason, K., Karlsson, J., Larsson, B., Lindroos, A. K., Lonroth, H., Naslund, I., Olbers, T., Stenlof, K., Torgerson, J., and Carlsson, L. M. 2009. Effects of bariatric surgery on cancer incidence in obese patients in Sweden (Swedish Obese Subjects Study): a prospective, controlled intervention trial. Lancet Oncol. 10: 653 - 662.

13. Sjostrom L., Lindroos, A., Peltonen, M., Torgerson, J., Bouchard, C., Carlsson, B., Dahlgren, S., Larsson, B., Narbro, K., Sjostrom, C. D., Sullivan, M., and Wedel, H. 2004. Lifestyle, diabetes, and cardiovascular risk factors 10 years after bariatric surgery. N Engl J Med. 351: 2683 - 2693.

14. Weickert M. O. and Pfeiffer, A. F. 2008. Metabolic effects of dietary fiber consumption and prevention of diabetes. J.Nutr. 138: 439 - 442.

15. Greenway F., O'Neil, C. E., Stewart, L., Rood, J., Keenan, M., and Martin, R. 2007. Fourteen weeks of treatment with Viscofiber increased fasting levels of glucagon-like peptide-1 and peptide-YY. J.Med.Food. 10: 720 - 724.

16. DiNicolantonio J. J., Bhutani, J., and O'Keefe, J. H. 2015. Acarbose: safe and effective for lowering postprandial hyperglycaemia and improving cardiovascular outcomes. Open.Heart. 2: e000327 - .

17. de Jonge C., Rensen, S. S., Verdam, F. J., Vincent, R. P., Bloom, S. R., Buurman, W. A., le Roux, C. W., Schaper, N. C., Bouvy, N. D., and Greve, J. W. 2013. Endoscopic duodenal-jejunal bypass liner rapidly improves type 2 diabetes. Obes.Surg. 23: 1354 1360.

18. Morishita M., Tanaka, T., Shida, T., and Takayama, K. 12-8-2008. Usefulness of colon targeted DHA and EPA as novel diabetes medications that promote intrinsic GLP-1 secretion. J.Control Release. 132: 99 - 104.

19. Dumoulin V., Moro, F., Barcelo, A., Dakka, T., and Cuber, J. C. 1998. Peptide YY, glucagon-like peptide-1, and neurotensin responses to luminal factors in the isolated vascularly perfused rat ileum. Endocrinology. 139: 3780 - 3786.

20. Tolhurst G., Zheng, Y., Parker, H. E., Habib, A. M., Reimann, F., and Gribble, F. M. 2011. Glutamine triggers and potentiates glucagon-like peptide-1 secretion by raising cytosolic Ca2+ and cAMP. Endocrinology. 152: 405 - 413.

21. Greenfield J. R., Farooqi, I. S., Keogh, J. M., Henning, E., Habib, A. M., Blackwood, A., Reimann, F., Holst, J. J., and Gribble, F. M. 2009. Oral glutamine increases circulating glucagon-like peptide 1, glucagon, and insulin concentrations in lean, obese, and type 2 diabetic subjects. Am.J.Clin.Nutr. 89: 106 - 113.

22. Plaisancie P., Dumoulin, V., Chayvialle, J. A., and Cuber, J. C. 1995. Luminal glucagonlike peptide-1(7-36) amide-releasing factors in the isolated vascularly perfused rat colon. J.Endocrinol. 145: 521 - 526.

23. Drucker D. J. 2005. Biologic actions and therapeutic potential of the proglucagon-derived peptides. Nat.Clin.Pract.Endocrinol.Metab. 1: 22 - 31.

24. Han X. S., Huang, Y., Jing, H. J., Zhang, A. W., Jiang, T., and Xu, Y. M. 2014. Neuroprotective effect of RYGB in Zucker fatty diabetic rats. Int.J.Clin.Exp.Med. 7: 3297 - 3304.

25. Thaler J. P. and Cummings, D. E. 2009. Minireview: Hormonal and metabolic 
mechanisms of diabetes remission after gastrointestinal surgery. Endocrinology. 150: $2518-2525$.

26. Sudre B., Broqua, P., White, R. B., Ashworth, D., Evans, D. M., Haigh, R., Junien, J. L., and Aubert, M. L. 2002. Chronic inhibition of circulating dipeptidyl peptidase IV by FE 999011 delays the occurrence of diabetes in male zucker diabetic fatty rats. Diabetes. 51: 1461 - 1469.

27. Gedulin B. R., Smith, P., Prickett, K. S., Tryon, M., Barnhill, S., Reynolds, J., Nielsen, L. L., Parkes, D. G., and Young, A. A. 2005. Dose-response for glycaemic and metabolic changes 28 days after single injection of long-acting release exenatide in diabetic fatty Zucker rats. Diabetologia. 48: 1380 - 1385.

28. Samocha-Bonet D., Wong, O., Synnott, E. L., Piyaratna, N., Douglas, A., Gribble, F. M., Holst, J. J., Chisholm, D. J., and Greenfield, J. R. 2011. Glutamine reduces postprandial glycemia and augments the glucagon-like peptide- 1 response in type 2 diabetes patients. J.Nutr. 141: 1233 - 1238.

29. Meek C. L., Lewis, H. B., Reimann, F., Gribble, F. M., and Park, A. J. 9-5-2015. The effect of bariatric surgery on gastrointestinal and pancreatic peptide hormones. Peptides.

30. Dechelotte P., Darmaun, D., Rongier, M., Hecketsweiler, B., Rigal, O., and Desjeux, J. F. 1991. Absorption and metabolic effects of enterally administered glutamine in humans. Am.J Physiol. 260: G677 - G682.

31. Samocha-Bonet D., Chisholm, D. J., Gribble, F. M., Coster, A. C., Carpenter, K. H., Jones, G. R., Holst, J. J., and Greenfield, J. R. 2014. Glycemic Effects and Safety of LGlutamine Supplementation with or without Sitagliptin in Type 2 Diabetes Patients-A Randomized Study. PLoS.One. 9: e113366 - .

32. Meek C. L., Reimann, F., Park, A. J., and Gribble, F. M. 2-26-2015. Can encapsulated glutamine increase GLP-1 secretion, improve glucose tolerance, and reduce meal size in healthy volunteers? A randomised, placebo-controlled, cross-over trial. Lancet. 385 Suppl 1: S68 - .

33. Meek C. L., Lewis, H. B., Vergese, B., Park, A., Reimann, F., and Gribble, F. 11-2-2015. The effect of encapsulated glutamine on gut peptide secretion in human volunteers. Peptides.

34. Elahi D., Galiatsatos, P., Rabiee, A., Salas-Carrillo, R., Vakilipour, A., Carlson, O. D., Angeli, F. S., Shannon, R. P., Egan, J. M., and Andersen, D. K. 8-15-2014. Mechanisms of type 2 diabetes resolution after Roux-en-Y gastric bypass. Surg.Obes.Relat Dis.

35. Chambers E. S., Viardot, A., Psichas, A., Morrison, D. J., Murphy, K. G., Zac-Varghese, S. E., MacDougall, K., Preston, T., Tedford, C., Finlayson, G. S., Blundell, J. E., Bell, J. D., Thomas, E. L., Mt-Isa, S., Ashby, D., Gibson, G. R., Kolida, S., Dhillo, W. S., Bloom, S. R., Morley, W., Clegg, S., and Frost, G. 12-10-2014. Effects of targeted delivery of propionate to the human colon on appetite regulation, body weight maintenance and adiposity in overweight adults. Gut.

36. Bock G., Dalla, M. C., Micheletto, F., Basu, R., Giesler, P. D., Laugen, J., Deacon, C. F., Holst, J. J., Toffolo, G., Cobelli, C., Rizza, R. A., and Vella, A. 2010. The effect of DPP4 inhibition with sitagliptin on incretin secretion and on fasting and postprandial glucose turnover in subjects with impaired fasting glucose. Clin.Endocrinol.(Oxf). 73: 189 - 196. 
37. Vahl T. P., Tauchi, M., Durler, T. S., Elfers, E. E., Fernandes, T. M., Bitner, R. D., Ellis, K. S., Woods, S. C., Seeley, R. J., Herman, J. P., and D'Alessio, D. A. 2007. Glucagonlike peptide-1 (GLP-1) receptors expressed on nerve terminals in the portal vein mediate the effects of endogenous GLP-1 on glucose tolerance in rats. Endocrinology. 148: 4965 $-4973$.

38. Hansen L., Deacon, C. F., Orskov, C., and Holst, J. J. 1999. Glucagon-like peptide-1-(736)amide is transformed to glucagon-like peptide-1-(9-36)amide by dipeptidyl peptidase IV in the capillaries supplying the L cells of the porcine intestine. Endocrinology. 140: 5356 - 5363.

39. Burcelin R., Da, C. A., Drucker, D., and Thorens, B. 2001. Glucose competence of the hepatoportal vein sensor requires the presence of an activated glucagon-like peptide-1 receptor. Diabetes. 50: 1720 - 1728.

40. D'Alessio D. A. 2011. What if gut hormones aren't really hormones: DPP-4 inhibition and local action of GLP-1 in the gastrointestinal tract. Endocrinology. 152: 2925 - 2926.

41. Deacon C. F., Johnsen, A. H., and Holst, J. J. 1995. Degradation of glucagon-like peptide1 by human plasma in vitro yields an N-terminally truncated peptide that is a major endogenous metabolite in vivo. J.Clin.Endocrinol.Metab. 80: 952 - 957.

42. Vahl T. P. and D'Alessio, D. A. 2004. Gut peptides in the treatment of diabetes mellitus. Expert.Opin.Investig.Drugs. 13: 177 - 188.

43. Hansen L., Hartmann, B., Bisgaard, T., Mineo, H., Jorgensen, P. N., and Holst, J. J. 2000. Somatostatin restrains the secretion of glucagon-like peptide-1 and -2 from isolated perfused porcine ileum. Am.J.Physiol Endocrinol.Metab. 278: E1010 - E1018.

44. Lu Y., Levin, G. V., and Donner, T. W. 2008. Tagatose, a new antidiabetic and obesity control drug. Diabetes Obes.Metab. 10: 109 - 134.

45. Plaisancie P., Dumoulin, V., Chayvialle, J. A., and Cuber, J. C. 1996. Luminal peptide YY-releasing factors in the isolated vascularly perfused rat colon. J.Endocrinol. 151: 421 - 429.

46. McCreight L. J., Bailey, C. J., and Pearson, E. R. 2016. Metformin and the gastrointestinal tract. Diabetologia. 59: 426 - 435.

47. Wu T., Zhao, B. R., Bound, M. J., Checklin, H. L., Bellon, M., Little, T. J., Young, R. L., Jones, K. L., Horowitz, M., and Rayner, C. K. 2012. Effects of different sweet preloads on incretin hormone secretion, gastric emptying, and postprandial glycemia in healthy humans. Am.J.Clin.Nutr. 95: 78 - 83.

48. Mentis N., Vardarli, I., Kothe, L. D., Holst, J. J., Deacon, C. F., Theodorakis, M., Meier, J. J., and Nauck, M. A. 2011. GIP does not potentiate the antidiabetic effects of GLP-1 in hyperglycemic patients with type 2 diabetes. Diabetes. 60: 1270 - 1276.

49. Chang J., Wu, T., Greenfield, J. R., Samocha-Bonet, D., Horowitz, M., and Rayner, C. K. 2013. Effects of intraduodenal glutamine on incretin hormone and insulin release, the glycemic response to an intraduodenal glucose infusion, and antropyloroduodenal motility in health and type 2 diabetes. Diabetes Care. 36: 2262 - 2265. 